\title{
Rapid assessment procedures to describe tobacco practices at sites managed by Indian tribes
}

\author{
Roberta L Hall, Cynthia A Viles, Edward Lichtenstein, Russell E Glasgow, \\ Kerri L Lopez
}

\begin{abstract}
Objective-To describe patterns of tobacco use and non-use in buildings managed by a sample of Northwest US Indian tribes, and at buildings of comparable local government offices in adjacent communities, at baseline (1991) and after half of the tribes had received an intervention aimed at upgrading tobacco control policies (1993).

Methods - Rapid assessment procedures consisting of an objective check list of tobacco related artefacts, detailed observations of tobacco practices in various settings, and conversations with local people concerning tobacco practices; comparison of observations made in 1991 and 1993.
\end{abstract}

Results-Tobacco related artefacts and decreased in indoor areas, particularly in official tribal offices. Locations that appear resistant to tobacco control in both Indian and non-Indian areas were outdoor porches and entry ways, parking lots, restaurants, bingo halls, casinos, and offices of natural resource businesses such as forest products.

Conclusions - Observations made in field visits provided investigators with a description of the context within which tobacco control by Indian tribal governments is expressed, and identified social environments where tobacco control has been effective and those that need additional attention.

Department of Anthropology, Oregon

State University,

Corvallis, Oregon,

USA

RL Hall

CA Viles

Oregon Research Institute, Eugene, Oregon, USA

E Lichtenstein

RE Glasgow

Northwest Portland Area Indian Health

Board, Portland,

Oregon, USA

KL Lopez

Correspondence to Roberta $L$ Hall, Department of Anthropology, Oregon State University, Corvallis, OR 97331, USA.

\section{Introduction}

Surveys indicating a high prevalence of tobacco use and epidemiological data on tobacco related diseases among American Indians have indicated a need for programmes to reduce tobacco use in Indian populations. ${ }^{1}$ In developing health intervention programmes, surveys and quantitative assessments are usually the procedures of choice but can be difficult to obtain, as well as being costly and time consuming. ${ }^{2} \mathrm{~A}$ detailed qualitative social acceptance of personal tobacco use cedures focus on a specific issue or problem and tween the two approaches. Consistently, rapid description of the setting provides additional insights and can improve the delivery of the programme during its implementation period. ${ }^{3}$ Such procedures contribute to programme evaluation and help to determine future directions for intervention projects. ${ }^{45}$ The observational methodology has also been used effectively to evaluate worksite compliance with local no-smoking legislation. ${ }^{6}$

Applied anthropologists have developed techniques known as "rapid assessment procedures" (RAP) to meet the need for timely qualitative descriptions of the research setting which can be used to assess the progress of the intervention programme. ${ }^{4}$ While traditional ethnographies (systematic field studies that record cultural behaviour) examine all facets of a community's culture, rapid assessment proemploy a mixture of objective descriptions and interviews. Rapid assessment procedures differ from quantitative assessments in emphasis, though specific elements may be shared beassessment procedures attempt to obtain and portray the viewpoint of the community (the insider's or "emic" viewpoint that provides the social rules that govern a culture ${ }^{7}$ ); include direct field observations; are eclectic or multidisciplinary in the type of data they examine; use selective or convenience interviews rather than a random sample; and make thematic summaries of findings rather than frequency/ quantitative summaries ${ }^{8}$. Furthermore, rapid assessment procedures usually represent part of a programme of assessment and are not the sole means of description or evaluation. The descriptive assessments of practices related to tobacco obtained and analysed in this study fit the RAP profile. On the premise that other tobacco control projects will find the RAP approach useful, this paper describes methods and findings in a sample of Northwestern United States Indian tribes which were the subjects of a tobacco policy intervention pro-

$=$

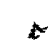
ject.

\section{Methods}

The rapid assessment procedure provided a description of the environments within which tobacco use by members of Northwest Indian tribes could occur; in this study tobacco use is limited to personal consumer use which has negative health implications and specifically excludes sacred or traditional use, which is 
considered a different category of behaviour. The principal goal of the rapid assessment procedures was to answer the question: Is this an environment conducive to personal tobacco use, or is it one in which such use is discouraged? Secondarily the assessments were designed to obtain a qualitative sense of attitudes and approaches related to tobacco control at the observed sites. The descriptive assessments were part of a five year tribal community intervention and consultation trial sponsored by the National Cancer Institute under a cooperative agreement with the Oregon Research Institute, the Northwest Portland Area Indian Health Board, and Oregon State University. ${ }^{9}$ The specific intervention offered to the federally recognised tribes of Washington, Idaho, and Oregon was culturally appropriate consultation toward the goal of developing or enhancing policies governing tobacco use within tribal buildings.

Following baseline assessment, the 39 federally recognised tribes in the Northwest were stratified by size, current status regarding tobacco policies, and cultural and geographical area, and then were randomly assigned to early intervention and late intervention groups. The consultation intervention lasted 18 months. Rather than being directed at smoking cessation, this project was directed at policy changes. Policies restricting tobacco use in tribally controlled buildings would have the immediate health effect of reducing environmental tobacco smoke but could also contribute to changes in social acceptance of personal tobacco use. ${ }^{9}$ Assessment, consisting of categorisation of tribal policies at baseline and after intervention, was based on interviews conducted with two tribal members knowledgeable about tribal tobacco policies. Detailed procedures and categories are presented elsewhere. ${ }^{10}$

Because funding limitations prohibited making descriptive assessments at all 39 sites, a subset of tribes was chosen to reflect the diversity of tribal conditions in respect to size, rural-urban settings, and other cultural and geographical characteristics. At the beginning of the study the Northwest tribes ranged in size from 124 to 7783 members. Seventeen tribes $(44 \%)$ had fewer than 1000 members and $22(56 \%)$ had more than 1000 members. Full time employees varied from eight to 874 , the tribes having large numbers of employees owning many businesses as well as operating a correspondingly large number of social service agencies. Tribes chosen for field visits had a similar demographic profile, varying in size from 250 to 7308 and representing a mix of rural and urban settings. Five of the $12(42 \%)$ had fewer than 1000 members and seven $(58 \%)$ had more than 1000 .

In the first round of visits in 1991, a single observer visited 11 tribes, six that were early intervention tribes and five in the late intervention mode. In the second round in 1993 the same observer visited 12 tribes, the original 11 plus an additional late intervention tribe. Two types of data were gathered: a checklist recording presence or absence of tobacco related artefacts (ash trays, cigarette stubs, nosmoking signs, anti-tobacco-use posters, etc) and a set of field notes recording observations of tobacco related behaviours and artefacts, which included reports of conversations with tribal members concerning tobacco issues. The observer, a college student and member of one of the Northwest tribes, had experience in working on cultural surveys and archives. When the observer entered a tribal office she identified her participation in the tribal tobacco policy project to tribal employees with whom she spoke and asked simply to look around.

A structured check list (table 1) was developed by staff of the tribal tobacco policy project and field tested at local government offices and businesses. The observer used the check list at each site visited, filling out a set of observations for each room or other type of space that she observed (a row in table 1). Completed records were given to the senior author who checked them for ambiguities, which were resolved before data were entered into a database program. The observer also made extensive field notes that described conversations concerning changing tobacco mores as well as observations of artefacts and behaviours related to tobacco control. Interviewees were selected on an ad hoc basis; they were primarily tribal employees and visitors at tribal offices who could offer insiders' views on changing tobacco practices. Checklists and field notes are the primary data analysed in this study.

Descriptive assessments were made at tribally owned sites, which included tribal government offices, health clinics, community centres, schools, and businesses. In addition, observations were made at non-Indian government buildings and public and commercial districts in adjacent communities that provide part of the social environment of tribal members. The observer spent an average of half a day with smaller tribes and up to 4.5 days with larger tribes, which maintain offices in several cities. The specific number of observed places (each occupying a row on the check list in table 1) varied from three to 51 per tribe in the first set of observations in 1991 and from eight to 48 per tribe in the second set in 1993. Tribes were sampled in proportion to their size and activities, but more checklists were completed in 1993 than in 1991-653 locations in 1993 compared with 382 in 1991. In both visits the number of observations correlated highly with tribal membership ( $\mathrm{r}=$ 0.745 for 1991 data and 0.894 for 1993 data) and with number of employees $(r=0.863$ in 1991 and 0.893 in 1993).

Every attempt was made to observe the same types of locations at each tribe: offices, agencies, businesses, and community centres, for example. A standardised protocol of visitation locations was not possible, however, given the great diversity among tribes. ${ }^{10} \mathrm{Ob}-$ servation strategies had to be adapted to the individual tribe's situation. For example, while some of the Northwest tribes have large reservations where tribal members predominate, other tribes have offices in cities that are 
Table 1 Check list of observations concerning tobacco

\begin{tabular}{|c|c|c|c|c|c|c|c|c|}
\hline \multirow{2}{*}{\multicolumn{4}{|c|}{$\begin{array}{l}\text { Name of Tribe (or Government) } \\
\text { Date } \\
\text { Observer }\end{array}$}} & \multirow[b]{3}{*}{$\begin{array}{l}\text { Evidence of } \\
\text { Educational } \\
\text { or Anti- } \\
\text { Tobacco } \\
\text { Activities }\end{array}$} & \multirow[b]{3}{*}{$\begin{array}{l}\text { Someone } \\
\text { Using } \\
\text { Tobacco } \\
\text { (specify } \\
\text { type) }\end{array}$} & \multirow[b]{3}{*}{$\begin{array}{l}\text { Coin-Op } \\
\text { Cigarette } \\
\text { Machines }\end{array}$} & \multirow[b]{3}{*}{$\begin{array}{l}\text { Cigarette } \\
\text { Stubs. } \\
\text { Tobacco } \\
\text { Cans or } \\
\text { Other } \\
\text { Tobacco } \\
\text { (specify } \\
\text { type) }\end{array}$} & \multirow[b]{3}{*}{$\begin{array}{l}\text { Number of } \\
\text { Minutes in } \\
\text { Area }\end{array}$} \\
\hline & & & & & & & & \\
\hline TRIBAL OFFICE (OR OTHER) & $\begin{array}{l}\text { No Smoking } \\
\text { Signs }\end{array}$ & Ashtrays & $\begin{array}{l}\text { Tobacco } \\
\text { Awareness } \\
\text { Posters }\end{array}$ & & & & & \\
\hline \multicolumn{9}{|l|}{ Entry-Outdoors } \\
\hline \multicolumn{9}{|l|}{ Entry-Indoors } \\
\hline \multicolumn{9}{|l|}{ Reception area } \\
\hline \multicolumn{9}{|l|}{ Meeting room } \\
\hline \multicolumn{9}{|l|}{ Conference room } \\
\hline \multicolumn{9}{|l|}{ Staff office } \\
\hline \multicolumn{9}{|l|}{ Tribal Health Office } \\
\hline \multicolumn{9}{|l|}{ Other (specify) } \\
\hline \multicolumn{9}{|l|}{ Parking lot } \\
\hline \multicolumn{9}{|l|}{$\begin{array}{l}\text { OTHER TRIBAL BUILDING } \\
\text { (Specify room type and building) }\end{array}$} \\
\hline Parking lot & & & & & & & & \\
\hline
\end{tabular}

In making observations, put + if you saw the item, and $O$ if you did not see it.

If you were not in the room or the building or if the tribe does not have a room or building of that type, put $X$ under "Number of minutes in Area."

Other notes:

Table 2 Locations where cigarette stubs or other tobacco refuse were observed ${ }^{a}$

\begin{tabular}{lccccc}
\hline & \multicolumn{2}{c}{ Tribal sites } & & \multicolumn{2}{c}{ Non-tribal sites } \\
\cline { 2 - 3 } Type of space & 1991 visit & 1993 visit & & 1991 visit & 1993 visit \\
\hline Indoor reception rooms & $6 / 30(20.0 \%)$ & $3 / 108(2.7 \%)$ & & $2 / 22(9.1 \%)$ & $2 / 75(2.7 \%)$ \\
Meeting rooms & $10 / 26(16.1 \%)$ & $11 / 90(12.2 \%)$ & & $4 / 34(11.8 \%)$ & $2 / 52(3.8 \%)$ \\
Staff offices & $1 / 27(3.7 \%)$ & $1 / 43(2.3 \%)$ & & $0 / 12(0 \%)$ & $0 / 25(0 \%)$ \\
Outdoor spaces & $75 / 90(83 \%)$ & $120 / 143(83.9 \%)$ & & $35 / 55(63.6 \%)$ & $92 / 105(87.6 \%)$ \\
\hline
\end{tabular}

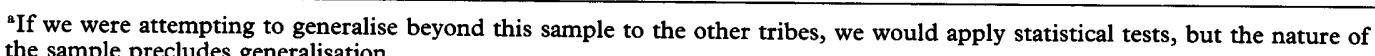
the sample precludes generalisation.

largely inhabited by the general population; some tribes operate public sector businesses, and others do not. The uniqueness of each tribal location precludes quantitative comparisons across tribes but permits qualitative comparisons within each tribe between observation periods. In addition to comparisons from the first visit to the second within each tribe, observations were compared between tribal environments and their non-tribal counterparts. While within tribe observations between periods are useful, generalisations cannot be made to the entire group of 39 tribes because the sample is too small and the observed tribes were not randomly chosen.

\section{Results}

Telephone interviews with key tribal contacts after the first round of interventions showed that the tribal tobacco policy project has had a positive impact upon policies regarding the use of tobacco within tribally controlled buildings. Analyses of these data appear elsewhere. ${ }^{1011}$ Field observations, the subject of this report, addressed the question of whether policy changes and attitudes have an observable counterpart in the social environ- ments to which policies apply. Rapid assessment strategies allowed us to ask whether tribal environments appeared to become less accepting of commercial tobacco use between the first, baseline, visit and the second visit 18 months later. The observations and conversations also gave insights into the processes of change and how they are perceived by tribal members and visitors at tribal offices.

For example, comparisons of checklist data forms found that more no-smoking signs were found in the offices of larger tribes than in the offices of small tribes. Field notes showed that this was due to size dependent differences in rules of social etiquette and the predominance of informal communication in smaller tribes. Informants at these tribes said that when a new policy was in effect most tribal members knew the rule and followed it, so signs were considered unnecessary or rude. For example, the manager of one of the smaller tribes told the observer that no signs were needed because all of the tribal members knew that tribal offices are smoke-free. In large tribes, however, formal communication was used. Field notes reported posters outside the entrance to offices at one of the larger tribes listing a series of punishable infractions, among them "smoking 
in unauthorised areas". Field notes describing observations of smoking in places where artefacts such as ash trays and cigarette stubs were seen confirmed that these artefacts are valid indicators of acceptance of tobacco use.

\section{QUANTITATIVE OBSERVATIONS}

In the baseline set of observations obtained at 11 sites, tobacco related artefacts in 382 different rooms (or other spaces indicated as rows on the check list in table 1) were recorded, 223 at tribal locations and 159 outside tribal control. In the second round at 12 sites the number of observed spaces increased to a total of 653 observations with 392 in tribal and 261 in non-tribal publically controlled areas. Table 2 categorises spaces that fall into four general types-indoor reception areas; conference rooms or general meeting rooms; private staff offices; and outdoor entry ways or parking lots - by year of observation and tribal or nontribal location. Listing raw numbers and the percentage of locations where tobacco artefacts were recorded, the table shows that tobacco artefacts were observed in a smaller proportion of indoor office spaces in 1993 than in 1991. Changes occurred both in tribal and non-tribal locations, but are more noticeable in tribal sites, where a higher percentage of sites recorded tobacco artefacts at baseline. Even in 1991, however, most tobacco use, both at Indian and non-Indian government offices, occurred outdoors; the proportion of outdoor locations where tobacco use was observed did not decrease in Indian sites and increased in non-tribal locations between 1991 and 1993

\section{QUALITATIVE OBSERVATIONS}

A principal contribution of the observation data is the identification of attitudes toward policies. While discussions with tribal employees at one site included comments equating freedom with smoker's rights, the vast majority of discussions with employees indicated an acceptance of changing norms on tobacco use indoors. Also reported were discussions with employees who said they complied with policies by taking smoke breaks outdoors. These were supported by behavioural observations; for example, field notes reported visitors to tribal and non-Indian government offices snuffing out cigarettes as they entered. A degree of reluctance to accept inevitable policy changes was seen in a sign at an employees' lounge. The sign on the door had been changed from "smoking permitted" to "no smoking permitted" by a felt marker pen, but the "no" was weak and somewhat inconspicuous. The presence of large standing ash trays at the outer entrance to buildings sometimes with explicit signs about "snuffing it out"-indicate the continuing use of tobacco, although usually not in proscribed areas. Exceptions to compliance include a situation in which the observer smelled cigarette smoke within an office building and traced it into a rest room, with the smoker remaining elusive. Although they signify infractions of rules, occasional reports of this nature indicate a keen awareness by smokers of policies prohibiting smoking. Much more commonly observed were instances of compliance, for example, smoking on porches and in parking lots; but both types of behaviours indicate awareness of tobacco control policies.

A second type of useful information provided by the qualitative assessments is the identification of the types of environments that have achieved tobacco control and of environments that still need attention. Descriptions given in field notes show that commercial buildings (such as restaurants and other businesses serving tourists) are most accepting of tobacco use indoors, whether the commercial space is managed by an Indian tribe, a non-Indian government, or the private sector. Visitor's centres and community centres were next in laxity in tobacco control while tribal, county, and city offices are the most strict regarding tobacco use.

Qualitative examination of the observer's field notes show that, while tobacco use is still common among subjects of the intervention, tobacco control has increased between the two observation periods. These data do not provide information on quantity or frequency of use, but offer objective evidence in support of other data suggesting that policy changes have occurred. ${ }^{10}$

\section{BASELINE AND POSTINTERVENTION COMPARISONS}

The senior author examined the objective checklist data and the descriptive field notes and made a qualitative evaluation of tribal and non-tribal settings, comparing the two observation periods. Checklist data and field notes indicate that among both the six early and five late intervention tribes are some that initially had a moderate amount of tobacco control within their office space as well as several that had little or none. Because samples are small and each tribe has many unique demographic as well as historical features, the relative progress of each tribe can be compared only with itself. Results given below are $\frac{D}{0}$ grouped by early intervention or late intervention mode for descriptive purposes; stat- $\widehat{\sigma}$ istical comparisons between early intervention $N$ and late intervention samples are inappropriate $N$ because of small numbers and the lack of 0 random sampling.

Among the six early intervention tribes visited in 1991 and 1993, three showed improved tobacco control by having a smaller 0 percentage of stubs and ash trays in the office $\frac{D}{D}$ space indoors and by qualitative changes in tobacco use behaviour. Checklist data and $\varnothing$ qualitative observations suggest no change in the other three early intervention tribes in 8 respect to whether tobacco use was socially acceptable or not. Among the five late in-흥 tervention tribes which were observed both in $\supsetneq$ 1991 and 1993, three environments appeared to stay the same regarding acceptance of personal tobacco use, while two appeared to have increased their degree of control on tobacco use in the 18 month interval. 
Some limited secular trends appear in this tally. Overall, five of the 11 tribes appeared to have increased tobacco control while six stayed the same. The five that showed improvement included four tribes which initially showed a relatively high degree of tobacco control and one which appeared low in control (table 3). The six that appeared to stay the same between 1991 and 1993 represent a similar spectrum at baseline. Showing improvement across the 18 month period was thus not confined to a particular baseline status. The modest degree of improvement attained by tribes compares favourably to adjacent non-tribal environments. Among the 11 non-tribal public environments observed at two time points, four appeared to increase in control, six stayed the same, and one appeared to weaken in tobacco control-a finding not observed among any of the tribal environments. Comparisons between observed levels of control in tribal and adjacent non-tribal areas shows that in 1991, eight received the same score, while two tribes and one non-tribal area showed more control than its adjacent area. The pattern was similar in 1993; seven tribal and non-tribal adjacent areas received equivalent scores for control, while three tribal areas and two non-tribal areas scored higher than their opposite.

\section{COMPARISONS OF OBSERVATION ASSESSMENTS} WITH POLICY CHANGES

A composite score of stringency of tribal tobacco policy based on interviews with two tribal leaders at baseline in 1991 and after intervention in 1993 is compared with qualitative observation ratings in table 3 . Based on explicit criteria, the composite policy score summarises tribal policies regarding indoor office and meeting rooms, while the observational ratings provide a single score that attempts to summarise both checklist and qualitative observations. The policy and observational datasets were collected and analysed independently by team members who were

Table 3 Comparison of observational and policy assessments.

\begin{tabular}{lccccc}
\hline & \multicolumn{2}{c}{1991 scores } & & \multicolumn{2}{c}{1993 scores } \\
\cline { 2 - 3 } Tribes & Observation & Policy & & Observation & Policy \\
\hline Tribe A & 4 & 1 & 5 & 6 \\
Tribe B & 4 & 4 & 4 & 6 \\
Tribe C & 5 & 5 & 5 & 6 \\
Tribe D & 4 & 6 & 5 & 6 \\
Tribe E & No data & No data & 1 & 1 \\
Tribe F & 4 & 6 & 5 & 6 \\
Tribe G & 4 & 2 & 4 & 6 \\
Tribe H & 4 & 6 & 5 & 6 \\
Tribe I & 5 & 4 & 5 & 6 \\
Tribe J & 2 & 1 & 2 & 1 \\
Tribe K & 2 & 0 & 3 & 1 \\
Tribe L & 5 & 6 & 5 & 6
\end{tabular}

Tribes $\mathrm{A}$ to $\mathrm{E}$ have fewer than 1000 members while tribes $\mathrm{F}$ to $\mathrm{L}$ have more than 1000 . Observation ratings of tobacco control range from 1 (low) to 5 (moderately high) and are based on a qualitative evaluation of check list data and descriptive field notes. The composite policy score, a 7 point scale from 0 to 6 , is a summation based on classification of policies as smoke-free $(2)$, moderately restrictive $(1)$, or unrestricted $(0)$ in three areas: council meeting rooms, common work areas, and private offices. unaware of each other's findings until evaluations were completed.

While changes in behaviour may precede changes in policy, and installation of tobacco policy may occur gradually. Table 3 shows that the two features appear concordant. Spearman rank order correlations between the observation ratings and composite policy scores confirmed correspondence between the two sets of measures. For the 1991 data, $r=0.535$, with a probability of $0.044(n=11)$ and for the 1993 scores $r=0.8411$, with an associated probability of $0.00(n=12)$.

Because each tribe controls a number of diverse settings where social rules differ, observational data cannot be reduced simply to a single score for each tribe. More useful generalisations can be made across tribes concerning the types of environments in which tobacco is likely to be tolerated and in which it is proscribed. In the 12 tribes observed in 1993, tobacco use or tobacco related artefacts were infrequently seen in tribal government offices; occasionally seen in community centres, restaurants, and natural resource businesses; and commonly seen in bingo parlors and casinos. Field notes indicate early stages of control even in bingo parlors and casinos, however, as most of those observed had non-smoking as well as smoking areas, and exerted other controls; for example, a sign at the entrance to a tribally run casino said "show courtesy, no pipes, no cigars."

\section{Discussion}

Tobacco use mores are rapidly changing in much of the country ${ }^{11}$ and may be expected to change in Indian tribes and in other Northwest communities. In this study, a rapid assessment procedure consisting of an objective checklist combined with qualitative observations and interviews provided insights into the social environments that the intervention on tobacco policy was trying to influence. At their most basic level, these observations confirmed that tobacco control is a genuine health issue in Indian populations and showed that policy changes by tribal authorities can have an impact on personal tobacco use within tribally controlled areas. Further, these observations at two points in time indicated some progress toward tobacco control by evidence of reduction in tobacco use within buildings. They also identified areas in which policy interventions have not been implemented. Official government offices under tribal control are most susceptible to policy changes enacted by tribal councils, while areas that are viewed as recreational as well as those that are associated with outdoor labour or natural resources are more resistant to tobacco control and may require other intervention techniques. While generally supportive of findings made by analysis of policies and the attitudes of tribal leaders, ${ }^{10}$ the observational data also confirm impressions of project interventionists that a great deal of personal tobacco use is still continuing among Indian populations of the 
Northwest and more work needs to be done to reduce the prevalence of tobacco use.

Examination of non-Indian areas by the same procedures as those applied in the target population suggested that considerable similarity exists between sites under Indian control and those controlled by the general population. In both areas the greatest amount of control exists in official government offices and the least in tourist and recreational areas.

The strength of this approach lies in its ability to provide direct information about the communities where an intervention takes place. This is especially important if - as in this project with Northwest US Indian tribes - the targeted population differs culturally from the dominant culture for which more extensive data on intervention strategies have been developed. Limitations involve the cost of travel to the field; in this study, funding allowed field visits and observations to $30 \%$ of 39 sites - too few to permit generalisations. Interobserver reliability could be a problem in collecting descriptive data; in this study, standardisation was provided by having the same observer collect data from all sites.

Rapid assessment procedures involving a mix of objective checklists and detailed observations complement quantitatively oriented surveys and process data used to evaluate the effectiveness of the tobacco policy interventions. These rapid assessment procedures were applied in non-tribal as well as in tribal environments and could be adapted for other tobacco control interventions when a description of the environment in which tobacco control is being instituted is needed. While quantitative assessments are most appropriate to determine whether an intervention has obtained a specific effect, qualitative assessments provide descriptive data and the subjects' perspectives of the field situation that can help interpret results of an intervention and determine what directions programmes should take.

This study was supported by grant No U01 CA52230 from the National Cancer Institute. We are grateful for insights provided by Dr John Young concerning rapid assessment procedures used by applied anthropologists and for helpful comment made on an earlier draft of this paper by Dr Mac Marshall and by anonymous reviewers.

1 Burhansstipanov L, Dresser CM. Documentation of the cancer research needs of American Indians and Alaska natives. US Department of Health and Human Services, Public Health Service, National Institutes of Health, Public Health Service, National Institutes of Health, National

2 Scrimshaw NS, Gleason GR. Introduction. In: Scrimshaw NS, Gleason GR, eds. Rapid assessment procedures. Qualitative methodologies for planning and evaluation of health related programs. Boston: International Nutrition Foundation for Developing Countries, 1992: 1-8.

3 Kresno S, Garrison GG, Sutrisna B, Reingold A. Acute respiratory illnesses in children under five years in Indramaya, West Java, Indonesia: a rapid ethnographic assessment. Med Anthropol 1994; 15: 425-34.

4 Scrimshaw NS, Gleason GR, eds. Rapid assessment procedures. Qualitative methodologies for planning and evaluation of health related programs. Boston: International Nutrition Foundation for Developing Countries, 1992.

5 Jenkins C, Howard P. The use of ethnography and structured observations in the study of risk factors for the structured observations in the study of risk factors for the
transmission of diarrhea in highland Papua New Guinea Med Anthropol 1992;15:1-16.

6 Rigotti NA, Bourne D, Rosen A., Locke JA, Schelling TC. Workplace compliance with a no-smoking law: randomized community intervention trial. Am $\mathcal{F}$ Public Health 1992; 82: 229-35.

7 Bernard HR. Research methods in cultural anthropology. Newbury Park, California: Sage Publications, 1988: 226-7.

8 Afonja SA. Rapid assessment methodologies: application to health and nutrition programmes in Africa. In: Scrimshaw NS, Gleason GR, eds. Rapid assessment procedures. Oualitative methodologies for planning and evaluation of health related programs. Boston: International Nutrition health related programs. Boston: International Nutrition

9 Glasgow RE, Lichtenstein E, Hall R, Meyers GB, Wilder D, McRae SG. Indoor smoking policies of Indian tribe in the northwestern United States. Tobacco Control $1993 ; 2$ : 35-7.

10 Lichtenstein E, Glasgow RE, Lopez K, Hall R, McRae SG Myers GB. Promoting tobacco control policies in Northwest Indian tribes. Am $\mathcal{F}$ Public Health 1995; 85 991.

11 Rabin RL, Sugarman SD, eds. Smoking policy: law, politics, and culture. New York: Oxford University Press, 1993. 